\title{
NLFM 信号激励下非线性系统的最优共振响应研究*
}

\author{
宫涛 杨建华 ${ }^{\dagger}$ 单振 王志乐 刘后广
}

（中国矿业大学, 机电工程学院, 江苏省矿山机电装备重点实验室, 徐州 221116)

\section{摘 要}

非线性调频(NLFM)信号在雷达、通信、信号处理中应用广泛, 该类信号 所激励下的非线性系统响应有着丰富的信息, 通过共振来增强非线性调 频信号有一定实际意义与价值. 本文主要研究了受到不同类型 NLFM 信 号所激励的非线性系统共振现象, 提出了实时尺度变换方法来处理高频 的 NLFM 信号, 克服人为选择造成信号输出响应较差的缺点. 同时. 提出 了实时谱放大因子作为共振评价指标, 准确评价 NLFM 信号激瘀 的系 统共振响应, 讨论了系统参数对系统最优共振响应的影响, 参数选择在 合理的区间内即可实现最优共振响应, 不仅实现信号特征的大幅度增强, 还保持了信号时频特征的连续性. 最后, 将实时尺度变换方法和固定尺度 变换方法进行了对比, 说明了所提方法处理 NLFM 信号的优越性.

关键词: 系统共振, NLFM 信号, 信号增强, 实时㞋度变换

PACS : 05.45.-a, 43.60.Wy, 33.20.Tp, 43.60. Hy.

基金: 国家自然科学基金(批准号：12072362)、江苏高校优势学科建设工程资助的课题. †通讯作者.E-mail: jianhuayang@cumt.edu.cn

第一作者.E-mail: gongtao@cumt.edu.cn

\section{1 引 言}

共振是系统响应中一种典型的动力学现象. 通常来讲, 对于简谐激励, 当外激 励频率接近于振动系统固看频率时会发生共振现象, 此时系统振动振幅会急剧增 强. 不同于传统意义的共振，随机共振(stochastic resonance, SR $)^{[1]}$ 、振动共振 (vibrational resonance, VR) ${ }^{[2]}$ 得到广泛研究, 利用其独特的动力学特性可以增强微 弱的信号. 其中随机共振是在微弱低频信号、噪声和非线性系统协同作用下使得 系统输出得到显著增强的一种动力学现象, 最初由意大利学者 Benzi 提出 ${ }^{[3]}$, 之 后在信号处理中得到广泛应用 ${ }^{[4-6]}$. 随机共振发生的基本条件是输入的微弱信号满 足小参数条件, 即信号频率远小于 1 . 而实际中的信号大部分不满足小参数条件, 为了实现大参数信号的随机共振, 一些尺度变换方法被引入, 比如: 二次采样 ${ }^{[7]}$, 
频移尺度变换 ${ }^{[8]}$, 归一化尺度变换 ${ }^{[9]}$, 普通尺度变换 ${ }^{[10]}$. 振动共振与随机共振类似, 区别是输入的噪声被高频辅助信号代替, 尺度变换方法与随机共振的方法一致. 而单个信号激励下的系统共振 ${ }^{[11]}$ 是指不需要辅助信号, 依靠系统参数变化实现系 统输出增强的一种现象, 该现象有一定实际意义与价值, 在信号增强方面, 无需 额外的高频辅助信号、噪声即可实现信号的增强, 操作方便, 实现过程简单.

在信号处理领域，利用共振现象来增强不同类型的信号具有独特的优势，相

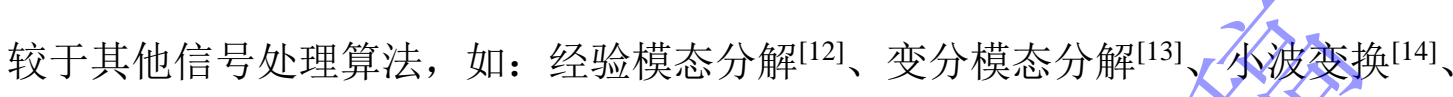
信号滤波 ${ }^{[15]}$ 等方法，该类方法不仅可以增强信号，而且可以有效抑制手扰噪声。 因此，基于共振的方法在信号处理中应用广泛. 许多学者利用最优共振现象来增

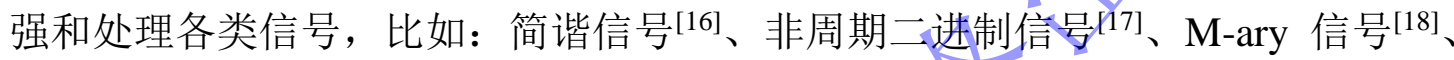

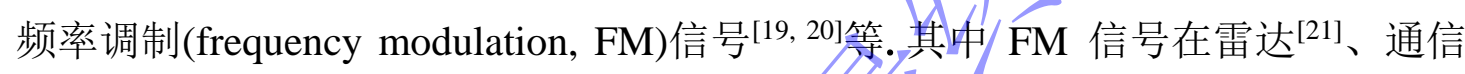

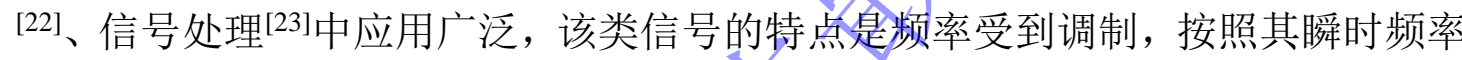
变化的规律主要分为线性调频(linear frequency modulation, LFM)信号和非线性调 频(nonlinear frequency modulation, NLFM)信号，目前已有一些 LFM 信号激励下 的非线性系统动力学行为的硑究, 但是针对 NLFM 信号的相关研究很少, 考虑到 NLFM 信号 ${ }^{[24-26]}$ 广泛存在, 如何实现 NLFM 信号最优共振增强效果及 NLFM 信 号激励下的非线性系统最优共振响应的研究是一个有实际意义与价值的问题.

目前关系系统共振的研究, 主要针对周期信号, 其信号频率固定, 通过尺度 变换方法即可满足共振的小参数要求, 调整合适的系统参数可实现最优共振响应. 但是对于复杂 NLFM 信号, 其频率随时间非线性变化, 此类信号的频率变化复 杂, 信号在频谱图中表现为连续频带, 而且之前所用的尺度变换方法没有考虑频 率的变化性, 采用傅里叶变换方法无法准确描述信号特征, 需要借助一些时频分 析方法来描述. 因此, 针对 NLFM 信号激励下的最优共振响应研究具有一定的难 
度与复杂性，采用的技术较周期信号的研究也更为复杂，采用固定尺度系数的尺 度变换方法来处理 NLFM 信号显然不能满足要求, 无法实现最优参数匹配, 系统 输出响应效果较差. 同时, NLFM 信号激励下的系统共振响应有一定的理论研究 价值, 对于实现 NLFM 信号增强有一定的意义. 基于此，本文研究了 NLFM 信号 激励下的非线性系统最优共振响应，提出了一种时变尺度系统共振方法有效实现 复杂 NLFM 信号的增强. 该方法的基本原理是利用实时变化的尺度系数来匹配瞬 时频率变化的 NLFM 信号和系统参数, 以尽量保证在每一时刻信号的瞬时频率满 足最优小参数条件，通过调整系统参数和尺度系数实现最优共振响应，

本文主要分为以下几部分进行研究: 第 2 部分提出了时变尺度系统共振理论, 同时定义了新的共振评价指标-实时谱放大因子，可有效评价 NLFM 信号的共振 增强效果; 第 3 部分处理了两类典型的 NLFM/信号, 研究了相关参数对最优共振 响应的影响，在保证信号时频特征连续性的同时, 实现了信号的共振增强, 与固 定尺度变换方法相比，本文所提方法克服又民度系数选择人为性造成信号增强效 果较差的缺点; 第 4 部分给出本文的主要结论.

\section{2 时变尺度系统共振理论}

一种时变双稳态系统荂振模型的动力学方程表示如下:

$$
\frac{\mathrm{d} x(t)}{\mathrm{d} t}=a(t) x(t)-b(t) x^{3}(t)+s(t),
$$

其中 $a(t) 、 b(t)$ 分别为时变系统参数 $(a(t) 、 b(t) \gg 1)$, 系统为双稳态系统. $x(t)$ 为系统 的输出, $s(t)$ 为输入的NLFM信号. NLFM信号 $s(t)$ 的瞬时频率 $f(t)$ 随时间不断变化, 为了实现尺度系数与信号 $s(t)$ 及系统参数 $a(t) 、 b(t)$ 相匹配, 引入了时变尺度系数 $\beta(t)$, 表达式为

$$
\beta(t)=\beta_{0} f(t),
$$

其中 $\beta_{0}$ 为尺度系数常数, $f(t)$ 为 $s(t)$ 的瞬时频率, $\beta(t)$ 满足条件 $\beta(t) \gg 1$. 同时, 系统 
参数 $a(t) 、 b(t)$ 与时变尺度系数 $\beta(t)$ 满足以下关系

$$
\frac{a(t)}{\beta(t)}=a_{1}, \frac{b(t)}{\beta(t)}=b_{1},
$$

引入变量替换 $\tau=\beta(t) t, x=z(\tau)$, 式(1)可写为

$$
\frac{\mathrm{d} z(\tau)}{\mathrm{d} \tau}=\frac{a(t)}{\beta(t)} z(\tau)-\frac{b(t)}{\beta(t)} z^{3}(\tau)+\frac{1}{\beta(t)} s\left(\frac{\tau}{\beta(t)}\right),
$$

将式(3)带入式(4)中, 有

$$
\frac{\mathrm{d} z(\tau)}{\mathrm{d} \tau}=a_{1} z(\tau)-b_{1} z^{3}(\tau)+\frac{1}{\beta(t)} s\left(\frac{\tau}{\beta(t)}\right)
$$

其中 $a_{1} \ll 1, b_{1} \ll 1$, 式(5)满足系统共振小参数条件. 式(5)相较式(1), NLFM信号 $s(t)$ 幅值缩小到 $1 / \beta(t)$, 将式(5)的NLFM信号幅值乘以 $\beta(t)$, 变为

$$
\frac{\mathrm{d} z(\tau)}{\mathrm{d} \tau}=a_{1} z(\tau)-b_{1} z^{3}(\tau)+s\left(\frac{\pi}{\beta(t)}\right)
$$

若系统共振发生在式(6)中，则系统共振也发生在式(7)中.

$$
\frac{\mathrm{d} x(t)}{\mathrm{d} t}=a(t) x(t)-b(t) x^{3}(t)+\beta(t) s(t)
$$

式(7)为一个大参数共振模型, 岢检测任意大频率的信号.

谱放大因子是经典的麿振性能评价指标, 针对NLFM信号, 信号的瞬时频率 非线性变化, 利用谱放大因子难以刻画NLFM信号的共振效果. 因此, 本文定义了 一种实时谱放大因子 $\eta$ 来刻画NLFM信号激励下的系统共振效果, 如下式

$$
\eta=\frac{1}{N} \sum_{i=1}^{N}\left[\frac{\frac{1}{(2 M+1)} \sum_{j=1}^{M}\left[X\left(f\left(t_{i}\right)-j\right)+X\left(f\left(t_{i}\right)+j\right)+X\left(f\left(t_{i}\right)\right)\right]}{S\left(f\left(t_{i}\right)\right)}\right],
$$

其中 $\eta$ 为系统输出的特征频带的放大程度, $N$ 为总数据点数, $M$ 为频率曲线 $f(t)$ 两侧 的频带宽度 $m$ 对应数据点数, $X(\cdot)$ 和 $S(\cdot)$ 分别为输入信号和输出信号的离散短时傅 里叶变换谱值, $f(t)$ 为 $t_{i}$ 时刻信号的瞬时频率. 核心是在STFT频谱上定义谱放大因 
子, 将输出信号STFT频谱图中信号时频一定范围内的能量之和与输入NLFM信号 的时频图中脊线能量之比作为评价指标，综合考虑NLFM信号的时频特征，描述 系统的共振响应更准确、可靠. 若 $\eta>1$, 说明NLFM信号被有效增强.

\section{3 几种不同NLFM信号增强仿真分析}

\section{1 分段NLFM信号}

分段 NLFM 信号较为典型，在机械设备信号处理中十分常见，由轴承、齿轮 等零件经过复杂路径产生的信号为 FM 信号, 按照转速变化的特征, 全采生反映 零件运行状态的 LFM 信号和 NLFM 信号. 其中与分段 NLFM 信豆频率特征相对 应的信号也很常见，比如文献 ${ }^{[27] 、}{ }^{[28]}$ 中这类转速工况，矿升提升机运行的典型转 速工况为速度曲线线性上升-平稳-线性下降, 反映出提升过程先线性加速-稳速线性减速, 这样的转速是人为控制的, 关键部位的轴承故障特征信号均为此类分 段 NLFM 信号; 流水线上的工业机器人的零件运行工况所产生的信号也为此类 NLFM 信号, 在故障诊断领域该类信号很常见，应用广泛，此类信号具有一定的 代表意义。

一种典型的分段 NLFM 信另 $\mathrm{S}_{1}(t)$ 的频率 $f(t)$ 随时间变化的分段函数为

$$
f(t)= \begin{cases}t_{0}+\gamma t & \left(0<t \leq t_{1}\right) \\ f_{0}+\gamma t_{1} & \left(t_{1}<t \leq t_{2}\right), \\ f_{0}+\gamma\left(t_{1}+t_{2}\right)-\gamma t & \left(t>t_{2}\right)\end{cases}
$$

其中 $f_{0}$ 为起始频率, $\gamma$ 为频率的变化率, $t_{1} 、 t_{2}$ 分别为频率规律发生变化的时刻. 对应余弦函数的 NLFM 信号 $s_{1}(t)$ 描述为 


$$
\begin{aligned}
s_{1}(t) & =A \cos \left[2 \pi \int_{0}^{t} f\left(t^{\prime}\right) \mathrm{d} t^{\prime}+\phi\right] \\
& =A \cos \left[\int_{0}^{t} 2 \pi\left\{\begin{array}{ll}
f_{0}+\gamma t^{\prime} & \left(0<t \leq t_{1}\right) \\
f_{0}+\gamma t_{1} & \left(t_{1}<t \leq t_{2}\right) \\
f_{0}+\gamma\left(t_{1}+t_{2}\right)-\gamma t^{\prime}+\phi & \left(t>t_{2}\right)
\end{array}\right],\right. \\
& =A \cos \left[2 \pi\left\{\begin{array}{ll}
f_{0} t+\frac{1}{2} \gamma t^{2} & \left(0<t \leq t_{1}\right) \\
\left(f_{0} t+\gamma t_{1} t\right) & \left(t_{1}<t \leq t_{2}\right)+\phi \\
{\left[f_{0}+\gamma\left(t_{1}+t_{2}\right)\right] t-\frac{1}{2} \gamma t^{2}} & \left(t>t_{2}\right)
\end{array}\right]\right.
\end{aligned}
$$

其中 $A$ 为信号幅值, $\phi$ 为初始相位, $s_{1}(t)$ 的频率变化规律为线性上升- 作变一线性下 降。

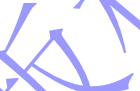

图 1 给出了该 NLFM 信号的相关信息, 图 1(a)和(b)分别为信号的时域波形 图和 STFT 频谱图, 在 STFT 频谱图中可以观察到信号的频率特征曲线在 $0 \sim 3 \mathrm{~s}$ 内从 $0 \mathrm{~Hz}$ 增加到 $150 \mathrm{~Hz}$, 然后保持至 $7 \mathrm{~s}$, 最后降到 $0 \mathrm{~Hz}$.
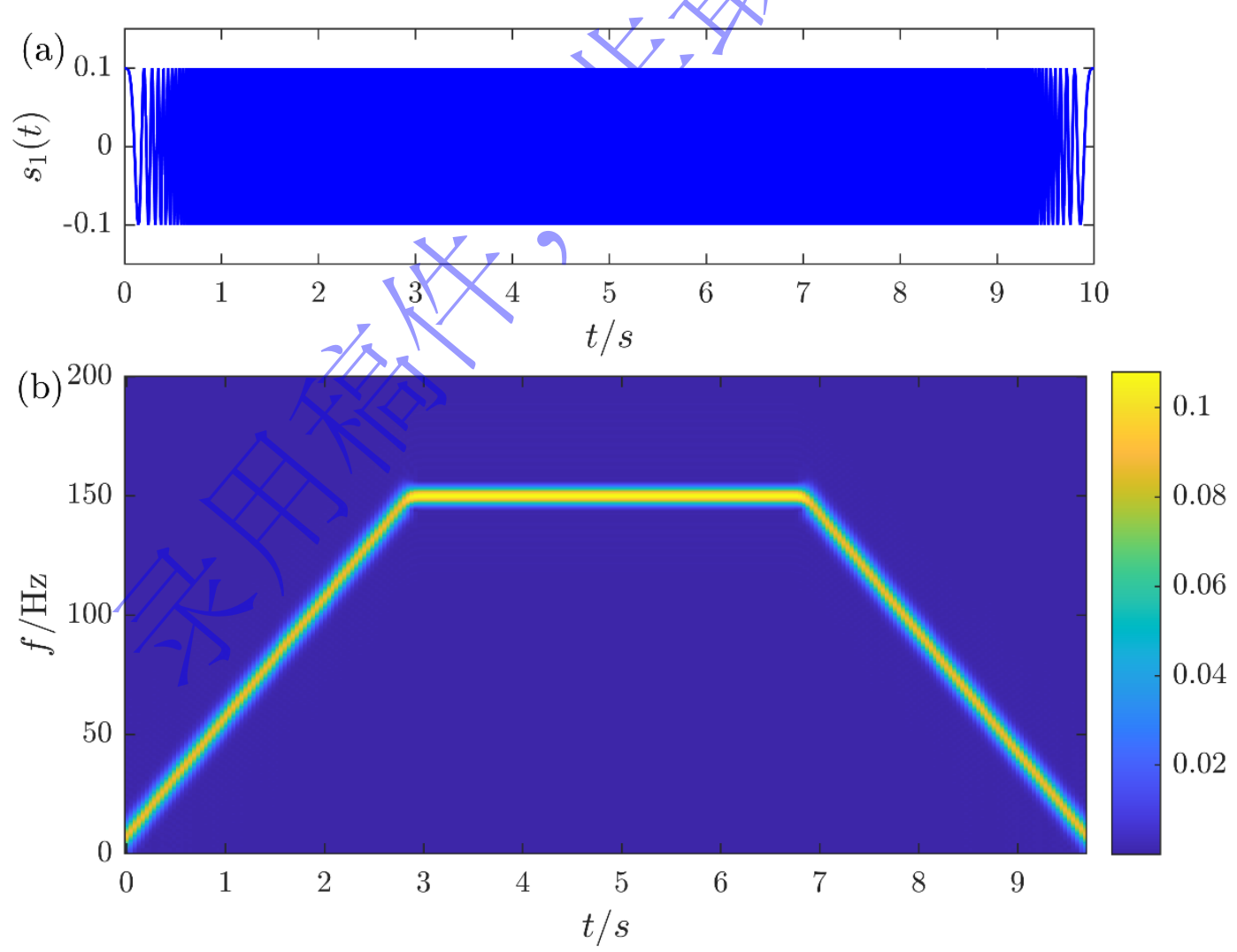

图 1 输入的 NLFM 信号 (a) 时域波形图; (b) STFT 频谱图 (仿真参数为 $A=0.1$, 
$\left.f_{0}=0, \gamma=50, \phi=0, t_{1}=3, t_{2}=10\right)$

Fig. 1. The input NLFM signal: (a) the time domain waveform; (b) the STFT spectrum. The simulation parameters are $A=0.1, f_{0}=0, \gamma=50, \phi=0, t_{1}=3, t_{2}=10$.

针对图 1 中的信号, 图 2 分别给出了不同尺度系数下的系统输出实时谱放大 因子 $\eta$ 随系统参数 $a_{1}$ 的变化曲线, 选取的固定的尺度系数有 $\beta=\beta_{0} \times 10, \beta_{0} \times 75$, $\beta_{0} \times 150$, 时变尺度系数有 $\beta=\beta_{0} \times f(t)$, 在固定尺度系数方法下的系统输出实时谱 放大因子 $\eta$ 随着尺度系数的增加而增强, 在最大值处实现了 NLFM 信夏最优系 统共振响应. 同时, 在时变尺度系数方法下的系统输出实时谱放灰子因最大值 $\eta_{\max }$ 比 $\beta=\beta_{0} \times 150$ 时的最大值略小, 但是区别不大.

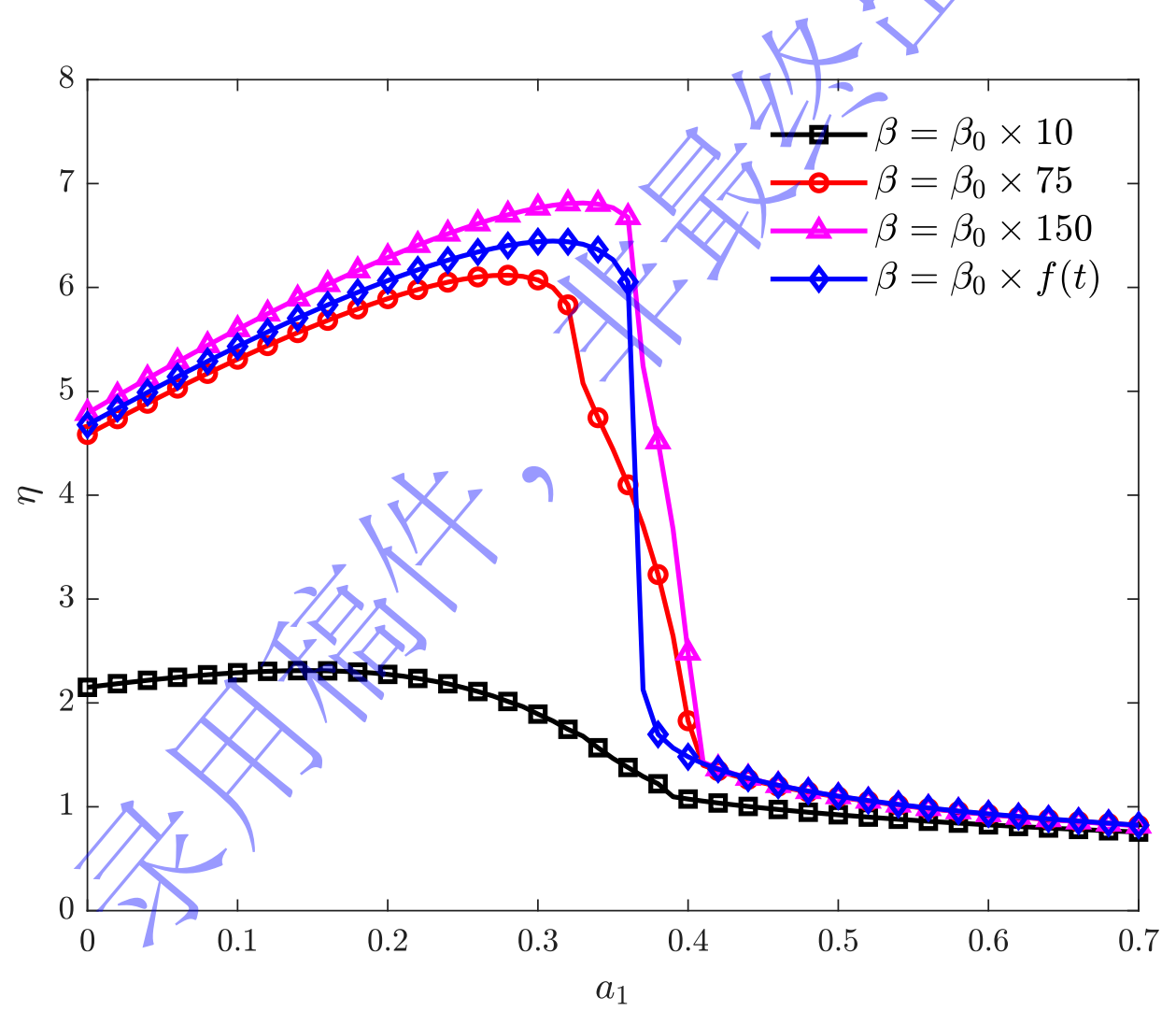

图 2 不同尺度系数 $\beta=\beta_{0} \times 10, \beta_{0} \times 75, \beta_{0} \times 150, \beta=\beta_{0} \times f(t)$ 下的系统参数 $a_{1}$ 与 实时谱放大因子 $\eta$ 的关系图 (仿真参数为 $b_{1}=1, \beta_{0}=100, m=2$ )

Fig. 2. Curves of $\eta$ - $a_{1}$ are obtained under different scale coefficient $\beta=\beta_{0} \times 10, \beta_{0} \times 75$, $\beta_{0} \times 150, \beta=\beta_{0} \times f(t)$. The simulation parameters are $b_{1}=1, \beta_{0}=100, m=2$. 
为了探究系统参数对最优共振响应的影响, 图 3 给出了实时谱放大因子 $\eta$ 与 系统参数 $a_{1} 、 b_{1}$ 的伪三维关系图. 其中 $\eta$ 数值的大小表示共振响应的强弱程度, 数值越大表示共振响应越强, 在图形中可以发现明显的共振区域, 在这个共振区 域内均可以发生系统最优共振响应, 实现 NLFM 信号增强.

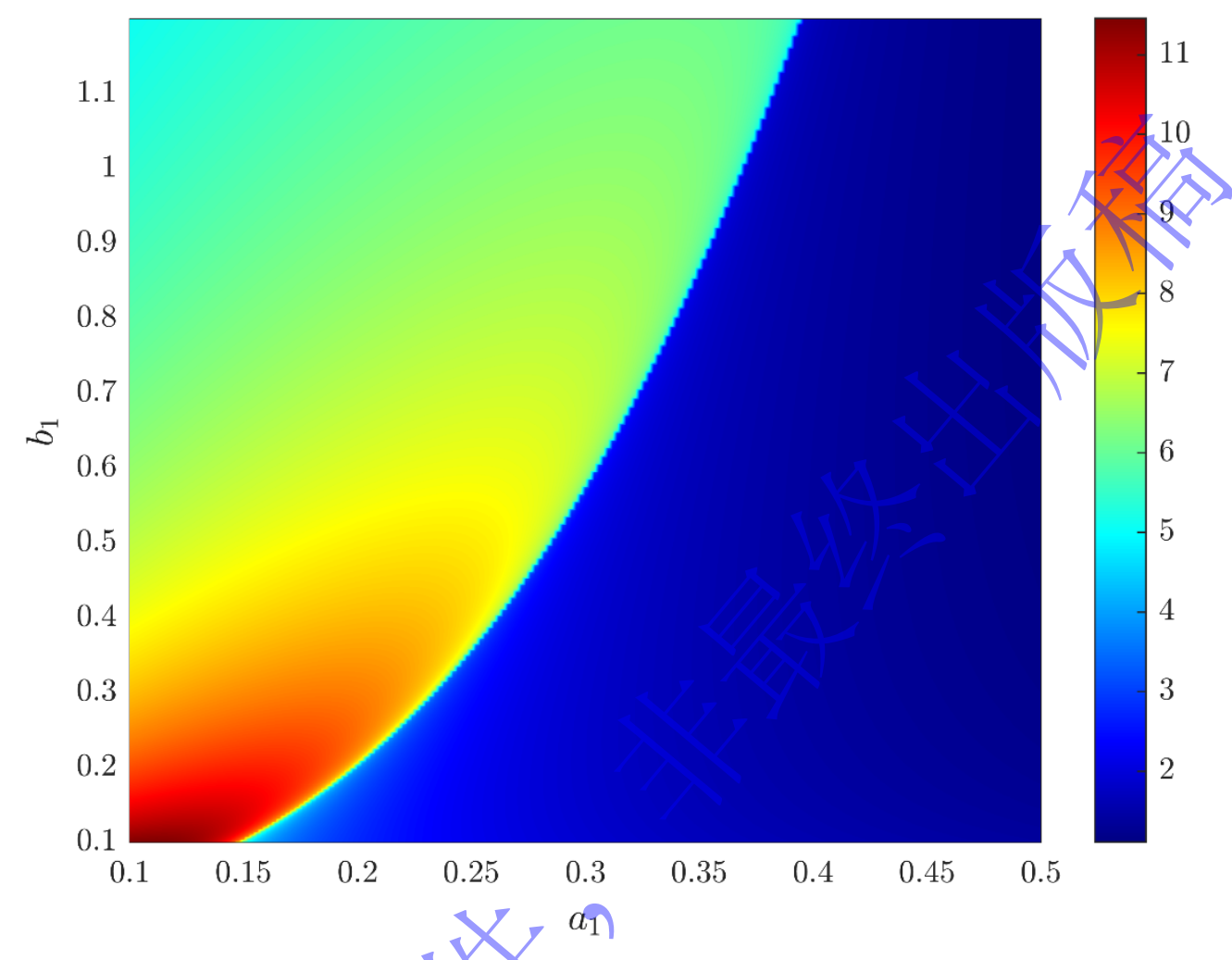

图 3 实时谱放大因子 $\eta$ 学系统参数 $a_{1} 、 b_{1}$ 的关系图 (仿真参数为 $m=2, \beta_{0}=100$ )

Fig. 3. Contour plot of the real time spectrum amplification factor $\eta$ in the $a_{1}-b_{1}$ plane.

The simulation parameters are $m=2, \beta_{0}=100$.

在图 2 禹 当 $a_{1}=0.32, b_{1}=1$ 时, 时变尺度方法对应的谱放大因子 $\eta$ 取得最 大值 $\eta_{\text {max }}=6.4$, 与其对应的最优系统共振响应如图 4 所示. 图 4 (a)和(b)分别为响 应的时域波形图和 STFT 频谱图, 信号幅值得到明显增强的同时保证了信号的连 续性. 

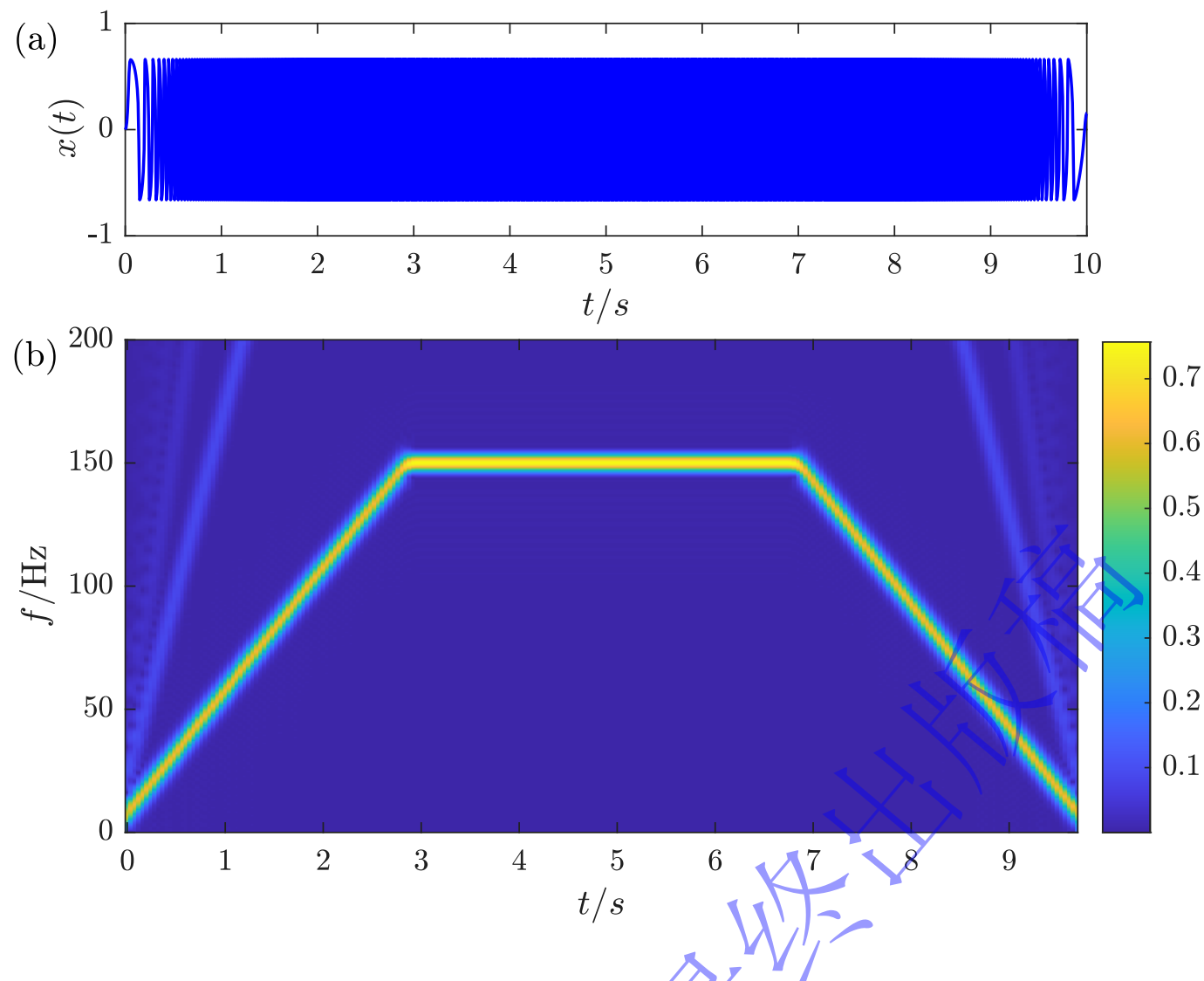

图 $4 b_{1}=1$ 时系统共振输出结果 (a) 时域波柇图, (b) STFT 频谱图 (仿真参数为 $\left.m=2, \beta_{0}=100\right)$

Fig. 4. The output response of system resonance when $b_{1}=1$ : (a) the time domain waveform; (b) the STFT spectrum. The simulation parameters are $m=2, \beta_{0}=100$.

为了说明时变尺度方法的必要性, 将固定尺度系数变尺度方法和时变尺度系 数变尺度方法的最优输出响应进行对比. 本文选择固定尺度系数为 $\beta=\beta_{0} \times 10, \beta_{0}$ $\times 75, \beta_{0} \times 150$, 目的是模拟尺度系数较小、适中、较大的情况，尺度变换后信号 的频率变为 $f(t) \times \beta$, 在图 2 中 $f(t)$ 的范围为 $0 \leq f(t) \leq 150$, 采用固定尺度系数变换 后频率范围分别为 $0 \leq f(t) /\left(\beta_{0} \times 10\right) \leq\left(15 / \beta_{0}\right), 0 \leq f(t) /\left(\beta_{0} \times 75\right) \leq\left(2 / \beta_{0}\right), 0 \leq f(t) /\left(\beta_{0} \times 150\right)$ $\leq\left(1 / \beta_{0}\right)$ ， 尺度系数基数 $\beta_{0}$ 固定（文中取 $100 ）$, 可以发现固定尺度系数为 $\beta_{0} \times 10$ 时, 尺度变换后频率达到 0.15 , 此时虽然满足系统共振小参数要求, 但是没有达 到最优输出效果 (见图 5), 说明尺度变换后的频率需要进一步降低; 当尺度系数 逐渐增大, 尺度变换后的频率逐渐减小，此时针对整个信号均可以满足共振的小 
参数要求, 实现最优系统共振响应.

图 5 为不同固定尺度系数 $\beta$ 下的最优系统共振响应结果，当 $\beta=\beta_{0} \times 10$ 时， 系统最优输出响应变得很差, 在图 5(a)中的信号幅值增强效果不显著, 图 5(b)的 时域波形图中信号波形产生了很大的失真. 当 $\beta$ 分别为 $\beta_{0} \times 75, \beta_{0} \times 150$ 时, 系统 共振达到最优输出响应, 与时变尺度方法下的最优输出响应区别不大. 但是, 固 定尺度系数下系统最优响应依赖于人为选择的尺度系数，一旦参数选择不理想， 共振输出响应会变得很差, 而时变尺度方法将系数定为 NLFM 信号的频率 $f(t)$, 克服了人为选择造成的系数最优输出响应不稳定的缺点.
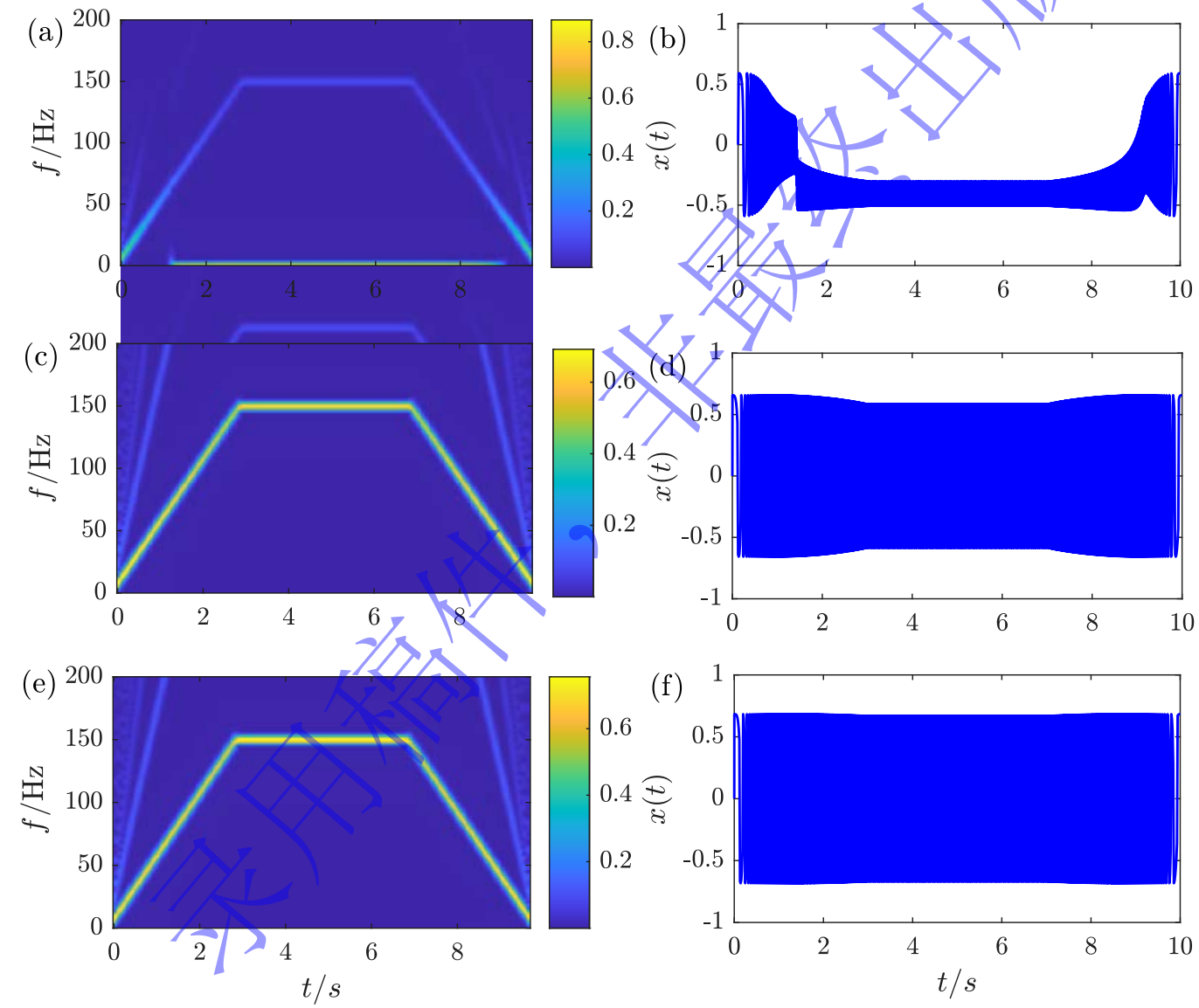

图 5 不同的固定尺度系数 $\beta$ 下的系统共振输出结果 (a) $\beta=\beta_{0} \times 10$ 下系统输出 STFT 谱图; (b) $\beta=\beta_{0} \times 10$ 下系统输出时域波形图; (c) $\beta=\beta_{0} \times 75$ 下系统输出 STFT 谱图; (d) $\beta=\beta_{0} \times 75$ 下时域波形图; (e) $\beta=\beta_{0} \times 150$ 下系统输出 STFT 谱图; (f) $\beta=$ $\beta_{0} \times 150$ 下时域波形图 (仿真参数为 $\beta_{0}=100$ ) 
Fig. 5. The output response of system resonance under different fixed scale coefficients $\beta$ : (a) the output STFT spectrum under $\beta=\beta_{0} \times 10$; (b) the output time domain waveform under $\beta=\beta_{0} \times 10$; (c) the output STFT spectrum under $\beta=\beta_{0} \times 75$; (d) the output time domain waveform under $\beta=\beta_{0} \times 75$; (e) the output STFT spectrum under $\beta=\beta_{0} \times 150$; (f) the output time domain waveform under $\beta=\beta_{0} \times 150$.

\section{2 调幅-调频NLFM信号}

在信号处理中，由正弦信号频率调制和幅值调制的信号也是 稆典型的 NLFM 信号, 该类信号特点是瞬时频率随时间波动, 变化规律复杂, 研究这类信 号最优系统共振响应及增强具有重要意义. 因此, 根据参考文献 ${ }^{[29]}$, 本文构造了 一种调幅-调频的 NLFM 仿真信号，如式(13)所示.

$$
s_{2}(t)=0.1\left(1+\frac{1}{3} \cos \frac{2 \pi t}{9}\right) \cos \left(80 \pi t+30 \sin \pi t+20 \cos \frac{5 \pi t}{3}\right) \text {, }
$$

该仿真信号受到正弦幅值、双正弦频率调制,y如图 6 所示. 图 6(a)和(b)分别为该 信号的时域波形图和 STFT 频谱图，不同时刻信号的幅值发生变化，频率也在变 化，频率分布在 $10 \sim 75 \mathrm{~Hz}$. 黑色实线为 NLFM 信号通过 Hilbert 变换相位提取的 瞬时频率曲线, 与时频特征曲线相一致. 对于此类 NLFM 信号而言，固定尺度变 换方法不适用.

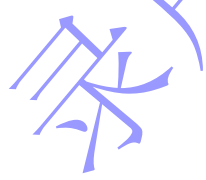



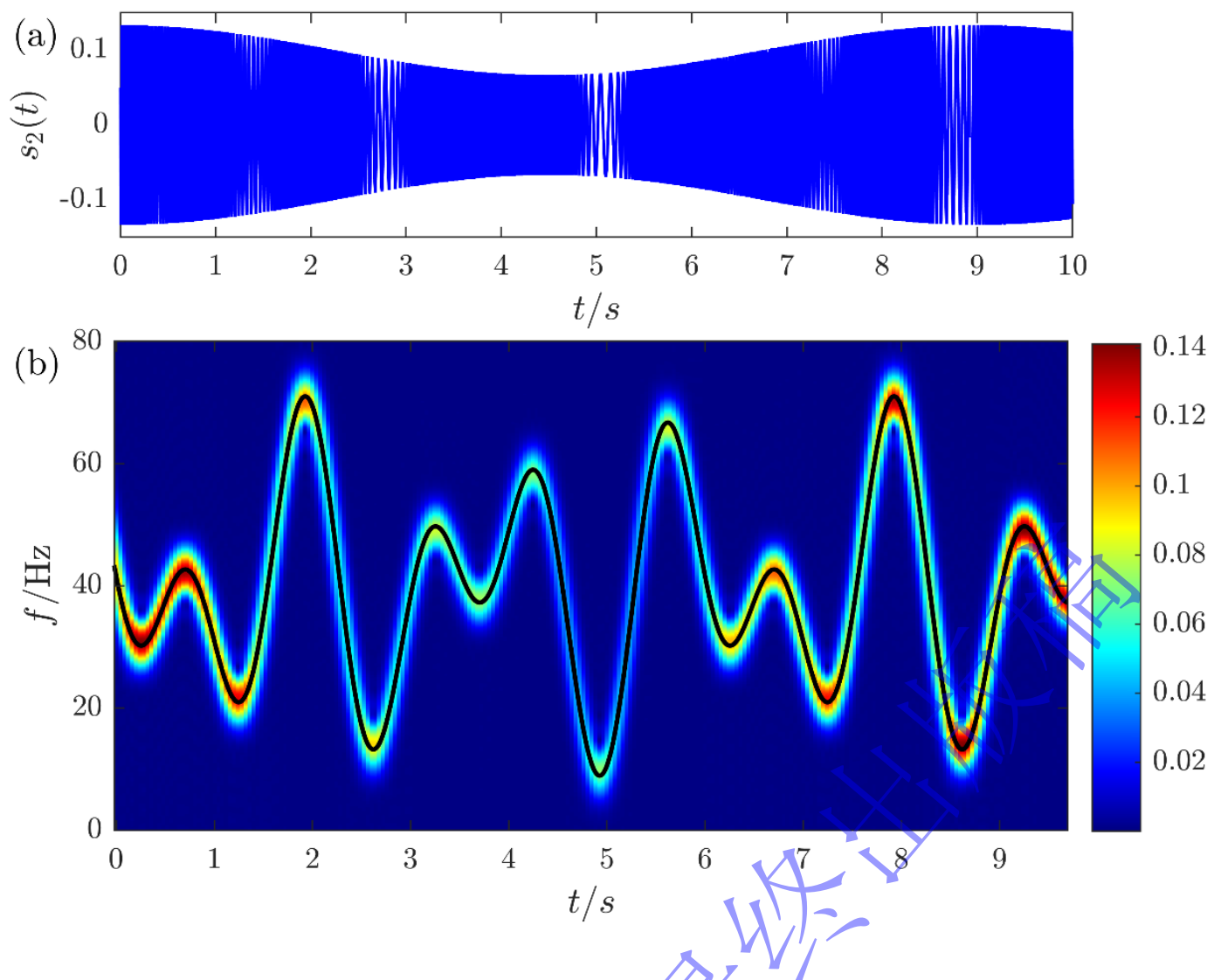

图 6 输入的 NLFM 信号 (a) 时域波形图: (b) STFT 时频图

Fig. 6. The input NLFM signal: (a) the time domain waveform; (b) the STFT spectrum.

图 7 为 $a_{1}=0.01$ 时不同尺度系数下实时谱放大因子 $\eta$ 与系统参数 $b_{1}$ 的关系 图, 在不同的尺度系数下, 随着 $b_{1}$ 的增大, $\eta$ 表现出先增加后减小的规律. 对于 时变尺度系数 $\beta=\beta_{0} \times f(t)$, 当 $b_{1}=0.0001, a_{1}=0.01$ 时, 实时谱放大因子最大为 101.1. 对于固定尺度系数的情况, 仅从数值上来看, 其谱放大因子既可能比对应 的实时尺度系数下共振输出结果小也可能比其大. 


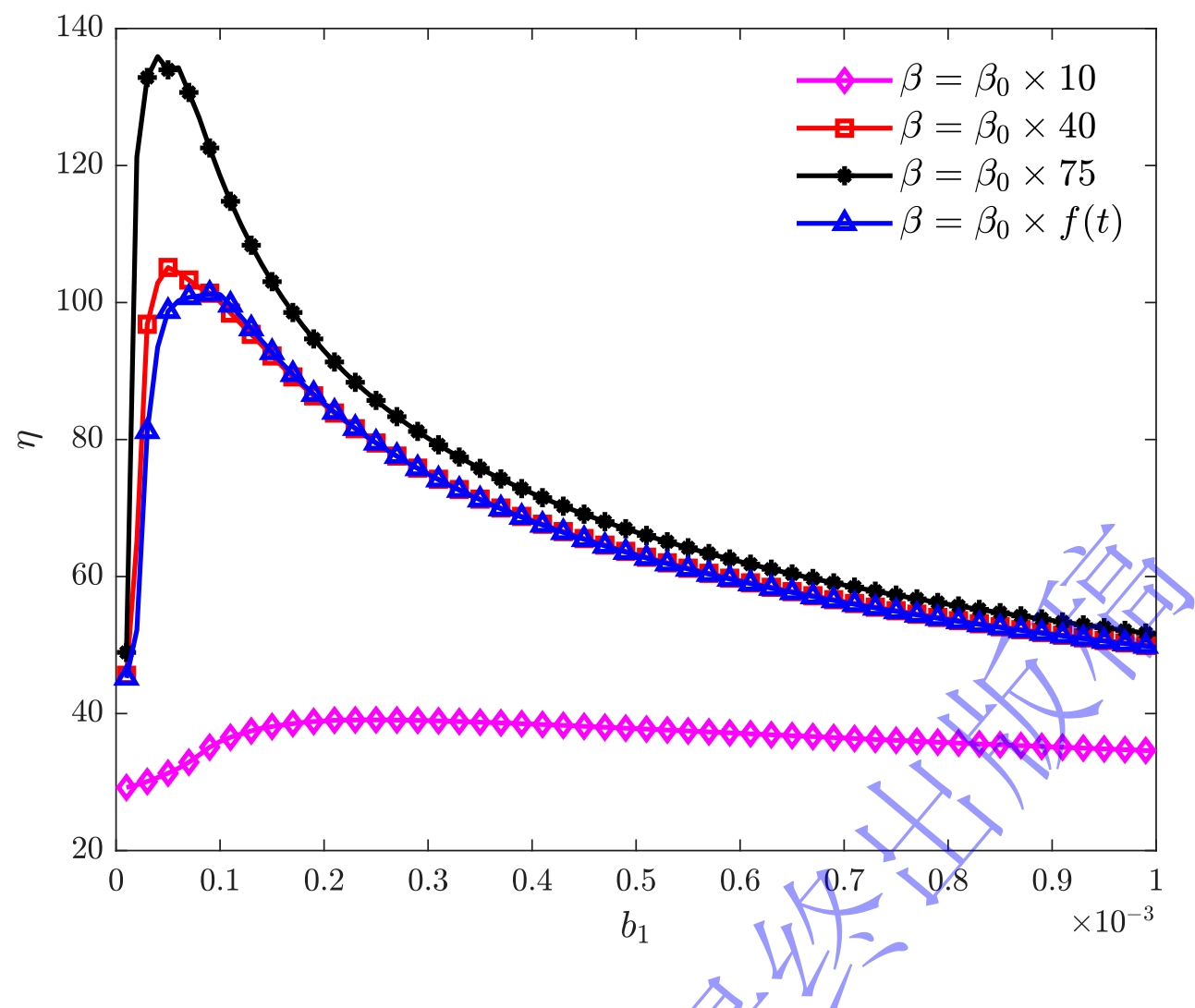

图 7 不同尺度系数下的实时谱放大因子 $\eta$ 与系统参数 $b_{1}$ 关系图 (仿真参数为 $a_{1}=$ $\left.0.01, m=5, \beta_{0}=1000\right)$

Fig. 7. Curves of $\eta-b_{1}$ are obtained under different scale coefficients. The simulation parameters are $a_{1}=0.01, m=5, \beta_{0}=1000$.

在时变尺度方法处理不, 其对应的最优输出信号如图 8 所示. 图 8 (a)和(b)分 别为最优系统共振响应时域波形图和 STFT 频谱图, 信号幅值得到大幅增强, 同 时保证信号时频特征的连续性. 需要说明的是在图 8(b)中短时傅里叶谱能量计 算范围，黑色实线包围区间表示响应信号的能量计算区间，该区间内所有 STFT 能量值之和作为输出谱能量, 输入信号谱能量为 NLFM 信号频率曲线上 STFT 能 量值之和. 

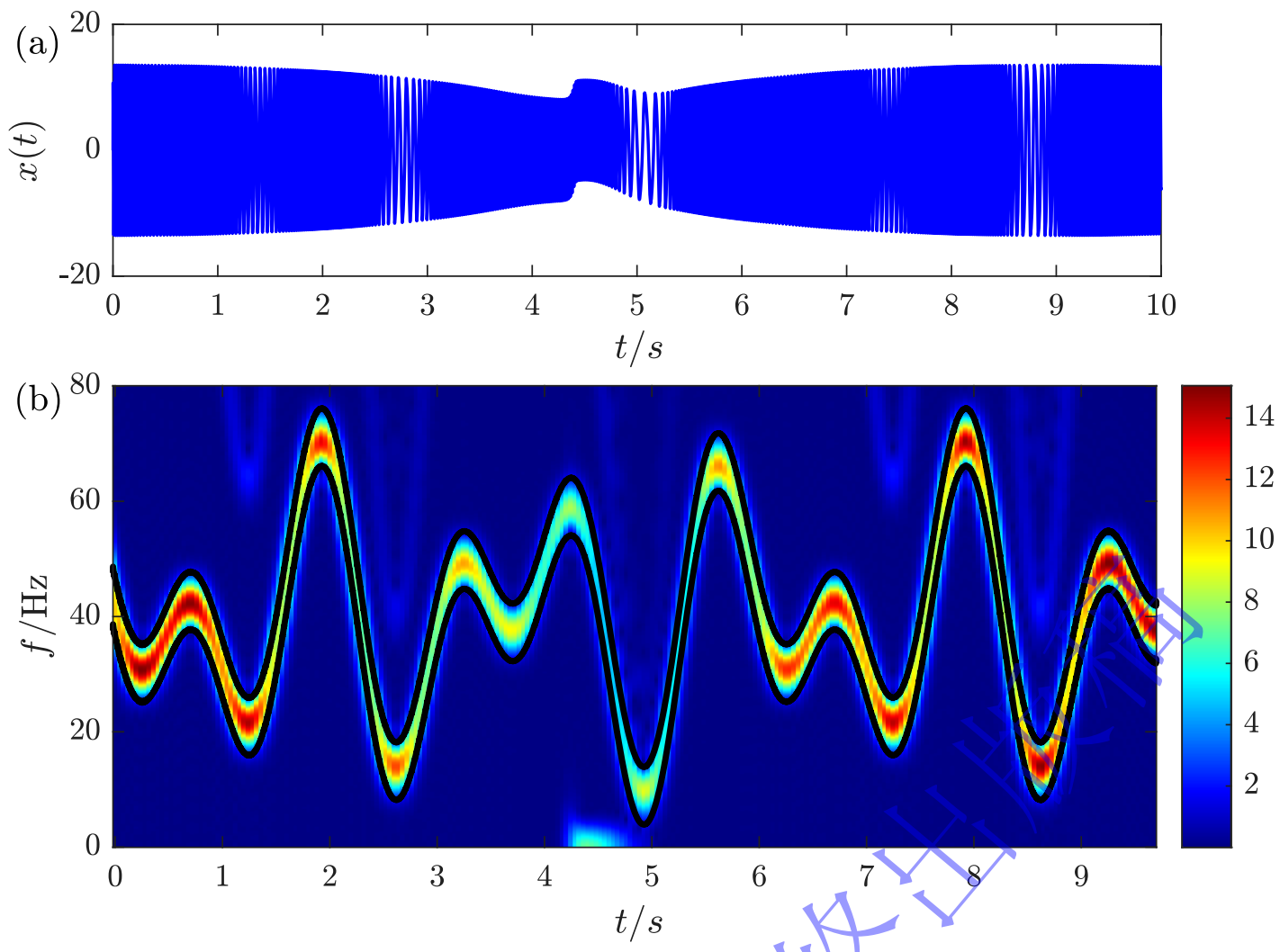

图 8 时变尺度系数下最优共振输出结果 (a) 时域波形图; (b) STFT 频谱图 (仿真 参数为 $\left.m=5, \beta_{0}=1000\right)$

Fig. 8. The output response of optimal resonance: (a) the time domain waveform; (b) the STFT spectrum. The simulation parameters are $m=5, \beta_{0}=1000$.

虽然在图 7 实时谱放大因 $3 \eta$ 与系统参数 $b_{1}$ 的关系图中，固定的尺度系数所 导致的共振结果 $\eta\left(\beta=\beta_{0} \times 40, \beta_{0} \times 75\right)$ 有的比时变尺度系数共振结果的 $\eta$ 要大一 些, 最优共振结果如图 9 所示, 但是固定尺度变换方法处理后的 NLFM 信号的连 续性很差, 能量发散现象严重, 远远没有时变尺度共振的效果好. 

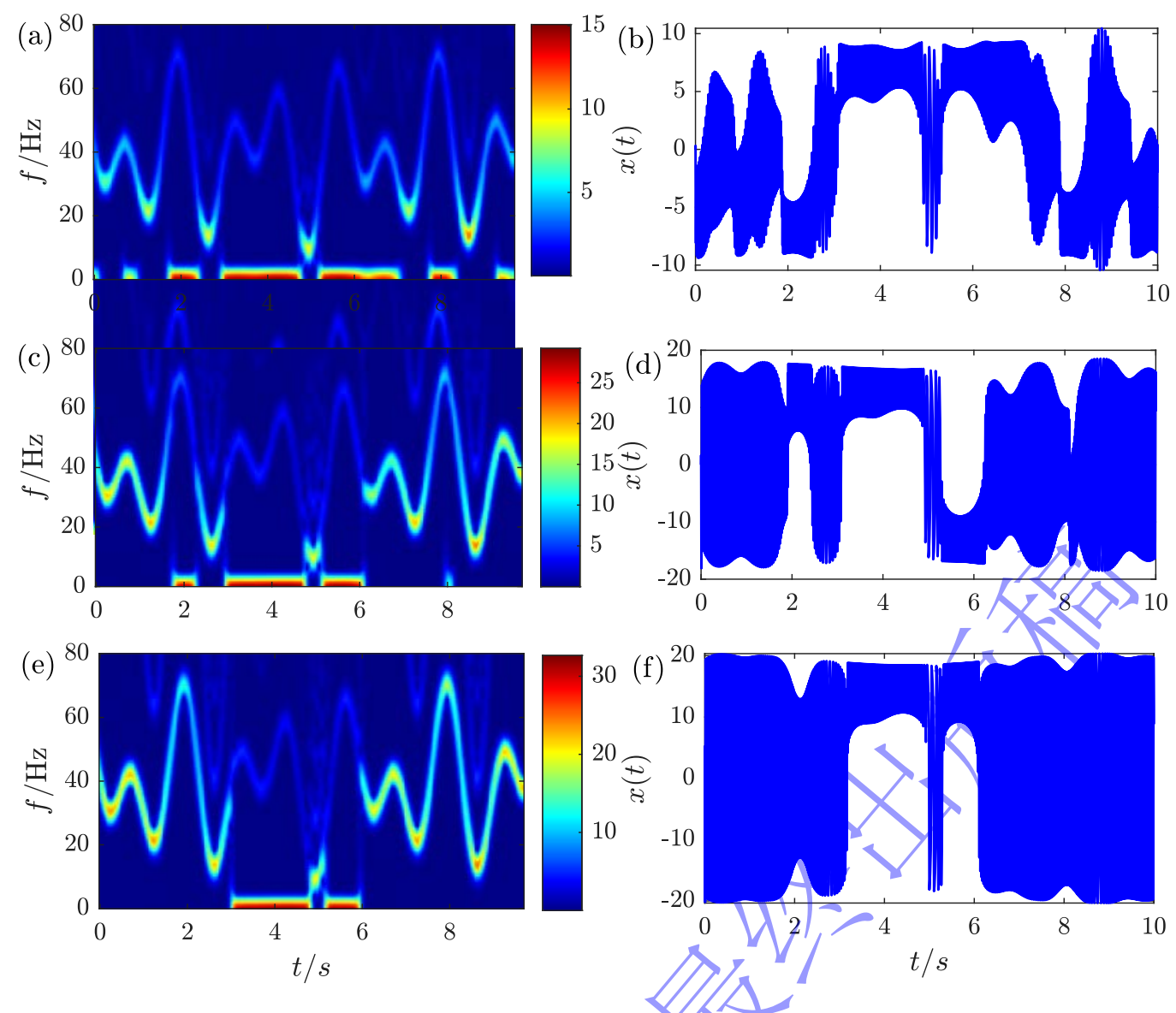

图 9 不同固定尺度系数 $\beta$ 下共振响应 (a) $\beta=\beta_{0} y 10$ 下输出 STFT 谱图; (b) $\beta=\beta_{0}$ $\times 10$ 下输出时域波形图; (c) $\beta=\beta_{0} \times 40$ 下输出 STFT 谱图; (d) $\beta=\beta_{0} \times 40$ 下输出时 域波形图; (e) $\beta=\beta_{0} \times 75$ 下输出 STFT 谱图; (f) $\beta=\beta_{0} \times 75$ 下输出时域波形图

Fig. 9. The resonance response under different fixed scale coefficient $\beta$ : (a) the output STFT spectrum under $\beta=\beta_{0} \times 10$; (b) the output time domain waveform under $\beta=\beta_{0} \times$ 10; (c) the output STFT spectrum under $\beta=\beta_{0} \times 40$; (d) the output time domain waveform under $\beta=\beta_{0} \times 40$; (e) the output STFT spectrum under $\beta=\beta_{0} \times 75$; (f) the output time domain waveform under $\beta=\beta_{0} \times 75$.

\section{4 结 论}

本文研究了两种 NLFM 信号激励下非线性系统最优共振响应现象, 主要结论 如下:

1) 针对传统的尺度变换方法不能匹配 NLFM 信号的时变频率特征的问题, 
提出了随信号瞬时频率变化而变化的时变尺度共振方法, 以实现每一时刻的最优 共振响应，进而增强 NLFM 信号.

2) 针对传统的共振效果评价指标-谱放大因子在 NLFM 信号激励下的系统共 振响应评价方面存在一定局限性，定义了新的指标-实时谱放大因子，可准确评价 NLFM 信号激励下系统共振响应, 同时保证了系统输出信号时频特征连续性.

3) 研究了非线性系统参数对共振输出响应的影响, 指出最优共振响应发生在 明显的共振带上，选择位于共振带的系统参数即可实现最优非线性系统委振输出， 实现对 NLFM 信号的增强.

本文实现了 NLFM 信号的最优非线性系统共振响应, 为 NLFM 信号增强及 检测提供参考, 在信号增强方面有一定的实际意义写价值.同时, 给出了 NLFM 信号激励下系统最优输出响应的相关影响规律, 对研究复杂信号激励下的系统动 力学行为有一定的参考价值.

\section{参考文献}

[1] Gammaitoni L, Hänggi P, Jung P, Marchesoni F 1998 Rev. Mod. Phys. 70223

[2] Landa P S, McClintock P VE E2000 J. Phys. A Math. Theor. 33 L433

[3] Benzi R, Sutera A, Nulpiani A 1981 J. Phys. A Math. Theor. 14 L453

[4] Wang S, Wang R Z 2018 Acta Phys. Sin. 67160502 (in Chinese) [王珊, 王辅忠 2018 物理学报 67 160502]

[5] Qiao Z, Lei Y, Li N 2019 Mech. Syst. Signal. Process. 122502

[6] Lu S, He Q, Wang J 2019 Mech. Syst. Signal. Process. 116230

[7] Leng Y G, Wang T Y 2003 Acta Phys. Sin. 522432 (in Chinese) [冷永刚，王太勇 2003 物理学报 52 2432]

[8] Tan J, Chen X, Wang J, Chen H, Cao H, Zi Y, He Z 2009 Mech. Syst. Signal. Process. 
[9] Hu N, Chen M, Qin G, Xia L, Pan Z, Feng Z 2009 Front. Mech. Eng. 4450

[10] Huang D, Yang J, Zhang J, Liu H 2018 Int. J. Mod. Phys. B 321850185

[11] Wu C, Yang J, Huang D, Liu H, Hu E 2019 Meas. Sci. Technol. 30035004

[12] Alsalah A, Holloway D, Mousavi M, Lavroff J 2021 Mech. Syst. Signal. Process.

151107385

[13] Kumar A, Zhou Y, Xiang J 2021 Measurement 168108402

[14] Sakar C O, Serbes G, Gunduz A, Tunc H C, Nizam H, Sakar B E, Apaydin H 2019 Appl. Soft Comput. 74255

[15] Wang G, Peng B, Feng Z, Yang X, Deng J, Wang N 2021 Signal Process. 179 107836

[16] Li Z, Chen B, Sun H, Liu G, Zhu S 2021 Chin. Phys. B 30080502

[17] Chizhevsky V N, Giacomelli G 2008 Phys. Rev. E 77051126

[18] Zeng L, Li J, Shi J 2012 Chaos Solitons Fractals 45378

[19] Peng H, Zhong S C, Tu Z, Ma H 2013 Acta Phys. Sin. 62080501 (in Chinese) [彭 皓，钟苏川，屠浙，鸟洪 2013 物理学报 62 080501]

[20] Yang J, Zhang S, Sanjuán M A F, Liu H 2020 Commun. Nonlinear Sci. Numer. Simul. 85105258

[21] Qu K, Zhang R F, Xiao P C 2021 Acta Phys. Sin. 70198402 (in Chinese) [屈奎, 张 荣福,肖鹏程 2021 物理学报 70 198402]

[22] Daskalakis S N, Kimionis J, Collado A, Goussetis G, Tentzeris M M, Georgiadis A 2017 IEEE Trans. Microw. Theory Tech. 655251

[23] Zhu D, Gao Q, Lu Y, Sun D 2020 Digit. Signal Process. 107102860 
[24] Alphonse S, Williamson G A 2021 IEEE T. Aero. Elec. Sys. 571793

[25] Song J, Gao Y, Gao D 2015 J. Commun. 10976

[26] Vizitiu I C 2014 Prog. Electromagn. Res. C 47119

[27] Kim Y, Park J, Na K, Yuan H, Youn B D, Kang C S 2020 Mech. Syst. Signal

Process. 138106544

[28] Li Y, Xu F 2021 Struct. Health. Monit. 14759217211033627

[29] Iatsenko D, McClintock P V E, Stefanovska A 2016 Signal Process. 125290

\title{
Study the optimal resonance response of a nonlinear
} system excited by NLFM signal

\author{
Gong Tao Yang Jian-Hua ${ }^{\dagger}$ Shan Zhen Wang Zhi-Le Liu Hou-Guang
}

(Jiangsu Key Laboratory of Mine Mechanical and Electrical Equipment, Sehool of Mechatronic Engineering, China University of Mining and Technology, Xuzhou 22116, China)

\section{Abstract}

Nonlinear frequency modulation (NLFM) signal is widely used in radar, communication and signal processing. The response of nonlinear system excited by this kind of signal has rich information. At the same time, enhancing different types of signals by resonance phenomenon has unique advantages in the field of signal processing. Compared with other signal processing methods, such as empirical mode decomposition (EMD), variational mode decomposition (VMD), wavelet transform (WT), signal filtering, etc., this kind of method can not only enhance the signal, but also effectively suppress the interference noise. Therefore, it has certain significance to study the nonlinear system optimal response excited by different NLFM signals and enhance the NLFM signal through resonance phenomenon. This paper mainly studies the nonlinearsystem resonance phenomenon excited by different NLFM signals, which is different from previous studies. Firstly, a real-time scale transformation method is proposed to process NLFM signals, its basic principle is to match different NLFM signals by real-time varying scale coefficients and system parameters. The signal frequency at each time corresponds to different scale coefficients and systêm parameters, so as to realize the optimal resonance response at each time. In order to describe the optimal resonance response excited by NLFM signal more accurately, different from the traditional spectral amplification factor, the real-time spectral amplification factor is introduced as the evaluation index. Then, the influence of system parameters on the optimal system resonance response is discussed and the optimal resonance region is obtained, which means the optimal resonance response can be achieved by selecting parameters in a reasonable range. This method not only greatly enhances the signal characteristics, but also maintains the continuity of signal time-frequency characteristics. Finally, the real-time scale transformation method is compared with the general scale transformation method, which shows the superiority of the proposed method in processing NLFM signal. The method and the results of this paper shows some potential in dealing with complex NLFM, which provides a reference for NLFM signal enhancement and detection, and has certain practical significance in signal enhancement. Furthermore, the relevant influence law of the system optimal response excited by the NLFM signal is 
given, which has a certain reference value for studying the system dynamic behavior under different signal excitations.

Keywords: System resonance, NLFM signal, Signal enhancement, Real-time scale transformation

* Project supported by the National Natural Science Foundation of China (Grant No. 12072362), and the Priority Academic Program Development of Jiangsu Higher Education Institutions.
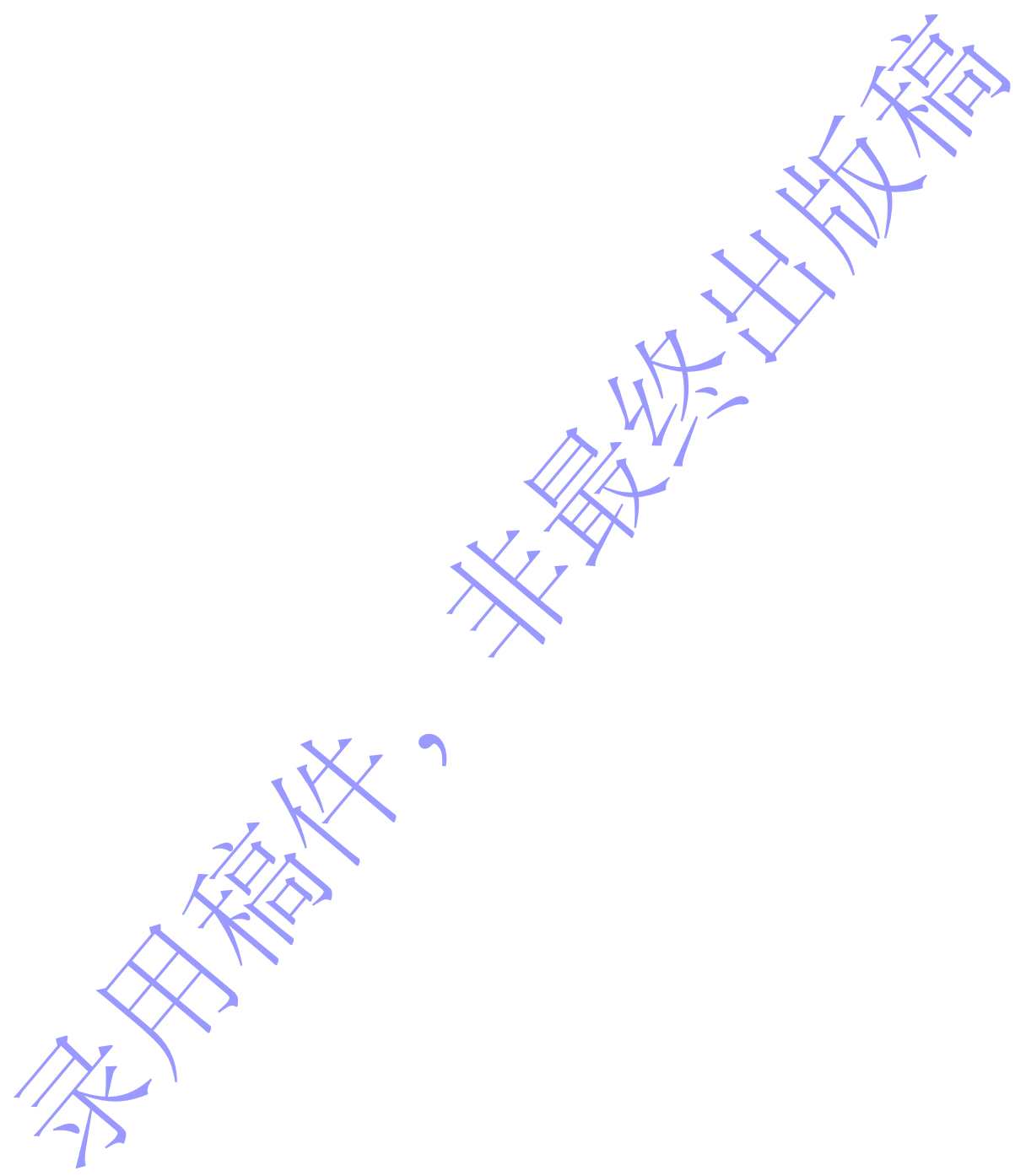